\title{
A Frequency offset estimation algorithms for OFDM mixed signals
}

\author{
Wei-cheng Xue $\mathrm{X}^{1,2,3, \mathrm{a}^{\star}}$, Changjiang Huang ${ }^{1}$, Jun $\mathrm{Ju}^{4}$ \\ ${ }^{1}$ National Time Service Center, the Chinese Academy of sciences, Xi'An, China \\ ${ }^{2}$ Graduate University of the Chinese Academy of Sciences, Beijing, China \\ ${ }^{3}$ Key Laboratory of Precision Navigation and Timing Technology, National Time Service Center, \\ Chinese Academy of Sciences, Xi'an, China \\ ${ }^{4}$ Jiangsu Broadcasting corporation \\ a420417514@qq.com
}

Keywords: OFDM, Composite frequency offset, fractional frequency offset, integral frequency offset

\begin{abstract}
The integer frequency offset and the fractional frequency offset estimation algorithms in SFN are improved and used for the Composite fractional frequency offset estimation in OFDM mixed signals in this paper。The simulation results show that the performance of the Composite frequency offset estimation is slightly lower than the single signal frequency offset estimation, but the estimation results are far less than the sub-carrier frequency interval of one percent. The simulation results also show that the composite frequency offset estimation algorithm can be used for the mixed - signal frequency offset estimation.
\end{abstract}

\section{Introduction}

Orthogonal Frequency Division Multiplexing (OFDM), which is a multi-carrier modulation, has been used in many digital communication systems such as streaming media broadcast, LTE and radar image transmission because of its high spectrum utilization rate and strong capability against frequency selective fading. However, OFDM technology is sensitive to the carrier frequency offsets and phase noise, so the incomplete match of the frequency between the receiver and the transmitter is easy to cause Inter Carrier Interference (ICI) and Inter Symbol Interference (ISI). In order to solve the problem of frequency offsets for OFDM systems, scientists and technologists have found some available algorithms such as methods based on pilot or training symbols, methods based on cyclic prefixes and methods based on OFDM signal or its spectrum characteristic[1,2]. These methods can nearly recover carrier frequency offset though fractional frequency offsets and integral frequency offsets estimation and achieve good results in different OFDM systems.

In the process of positioning by OFDM signals, the signals received by the receiver are some mixed signals mixed by some signals which come from different transmitter with different directions distances and have only different phase and amplitude, and the mixed signals are thoroughly same except for the phase and the delay. In these cases, a slow-moving receiver can ignore the Doppler frequency shifts and regard the signals coming from different transmitters as multi-path signals. In order to demodulate the received signals, the receiver estimate and recover the 'Composite frequency offset' of OFDM systems. In our OFDM frame structure, both exactly same synchronous signals are inserted in the frame header, so long as we use the two synchronous to estimate the fractional and integral frequency offset, we can restore the system carrier frequency. The synchronous signals are OFDM modulation signals based on PN sequences with good auto-correlation and cross-correlation performance[3].

In the existing algorithms, the fractional frequency offset can be estimated by the method of maximum likelihood (ML) with good estimation effect, and the residential frequency deviation is large smaller than sub-carrier frequency interval $1 \%$. There is no doubt that the method is an efficient and simple way for fractional frequency-offset estimation[4]. To achieve the integral frequency-offset estimation, we can correlate the local signal continuously increased or decreased one sub-carrier interval with received signal to acquire until the correlation peak appears. These 
algorithms are used in frequency offset estimation of OFDM mixed signals and verified by simulation in this paper[5].

\section{Composite fractional frequency-offset estimation}

In order to verify whether the maximum likelihood method can be used to obtain the complex frequency offset or not, we simulate the fractional frequency offset estimation algorithm. In simulation, it is supposed that the signal frequency of each base station is the same and the receiver is at a static state. In this way, the frequency offset between the base station and the receiver is exactly the same[6]. In simulation, the different weighted delayed signals are superposed into the composite signals, and the frequency offset values are calculated according to a certain algorithm, and then the residual frequency offset values are examined.

The simulation is based on the OFDM synchronization signal in the China Mobile multimedia broadcasting (CMMB) signal system, which can be used for the estimation of the fractional and integer frequency offset estimation[7,8]. Since the protection interval between symbols in signal system is $51.2 \mathrm{us}$, the maximum time delay of the multipath signal is within 512 sampling points in the $10 \mathrm{MHz}$ sample rate. It is assumed that the 3 or 4 signals are existed in the simulation, and time delays and amplitudes of the signal are different. Table2.1 lists simulation conditions of the representative composite frequency offset.

Table 2.1 simulation conditions of the representative composite frequency offset

\begin{tabular}{|c|c|c|c|}
\hline No. & $\begin{array}{c}\text { Amplitude } \\
\text { ratio }\end{array}$ & $\begin{array}{c}\text { Time delay } \\
\text { intervals }\end{array}$ & $\begin{array}{c}\text { Signals } \\
\text { number }\end{array}$ \\
\hline 1 & $2: 1: 1: 1$ & $100: 200: 200$ & 4 \\
\hline 2 & $1: 1.4: 1$ & $5: 5$ & 3 \\
\hline
\end{tabular}

\subsection{Composite frequency offset algorithm under large delay condition}

The No.1 signal in Table 2.1 is that four signals with different latency and amplitude are superposed to form a composite signal. We suppose that the amplitude ratio of different time-delay signals is 2:1:1:1 and the delay intervals are 100,200,200 sampling points, respectively. The correlated result of the different time-delay signals and the composite signal is shown in Figure 2. In the figure, the light gray curves represent the correlated values of different time-delay signals, and the black curve represents the correlated values of composite signals. To comparison, the amplitude is reduced by half.

As the picture shows, the position of the correlated peak of the composite signal is close to the strong signal, but near the peak value is no longer monotonous rising or falling, and the triangle correlated result is asymmetric.

We can see from the figure that the correlated result of the composite signal is still the triangle after the four different weighted signals are superposed, but the top of the triangle is smoother. At this time, the peak value will introduce large error if we still use the method of correlated peak to calculate fractional frequency offset, and the fractional frequency offset estimation error will be larger if the white noise is added. One feasible solution is to intercept some correlated values near the top of the relevant peak, and the fractional frequency offset estimation can be obtained by calculating the average value. 


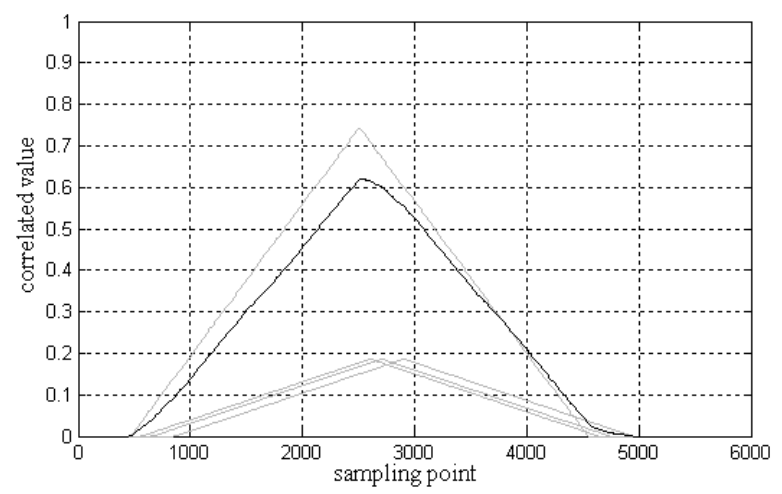

Fig.2.1 the correlated result of the composite signal

It is assumed that the maximum correlation peak value is $P_{k}$, so its absolute value is $\left|P_{k}\right|$. We choose the correlated values greater than $M \cdot\left|P_{k}\right|(0<M<1)$ as the data $P_{k n}$ which can be used to obtain the average value of the correlated peak, the number of these data is $\mathrm{N}$, the average value of the correlation peak is $\bar{P}_{k}$, then:

$$
\bar{P}_{k}=\sum_{1}^{N} P_{k n} / N
$$

At this time, the calculation formula of the fractional frequency offset can be transformed into:

$$
f=\frac{1}{2 \pi} \arctan \frac{\operatorname{Im}\left(\bar{P}_{k}\right)}{\operatorname{Re}\left(\bar{P}_{k}\right)} \quad(2-2)
$$

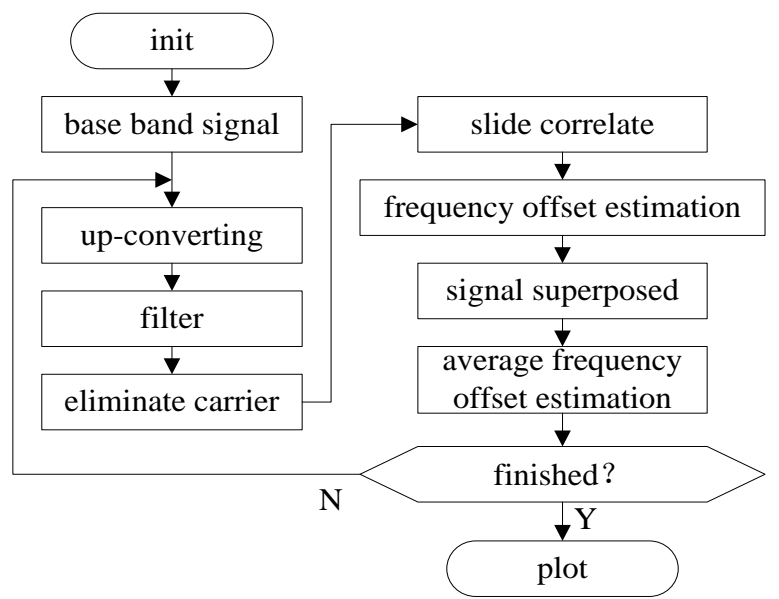

Fig.2.2 simulation process of the composite fractional frequency offset

In the simulation, the baseband signal is generated, and then the signal is modulated to $36 \mathrm{MHz}$ intermediate frequency signal which is added to a certain frequency offset, which simulate the received intermediate frequency signal, then we obtain the multipath signal by down-converting the intermediate frequency signal, and overlay them according to their respective delay. Then we slide the signal and correlate with the local synchronization signal, and then look for the relevant peak to calculate the frequency offset. The simulation process is shown in Fig.2.2.

At this time, the contrast result of the single signal and composite signal frequency offset is shown in Fig.2.3. In the figure, the horizontal coordinates are frequency-sweeping range, which represent the frequency offset from $-2400 \mathrm{~Hz}$ to $2400 \mathrm{~Hz}$, and the step of the sweeping frequency is $100 \mathrm{~Hz}$. The longitudinal coordinates are the frequency offset estimation errors, which units are Hz. 


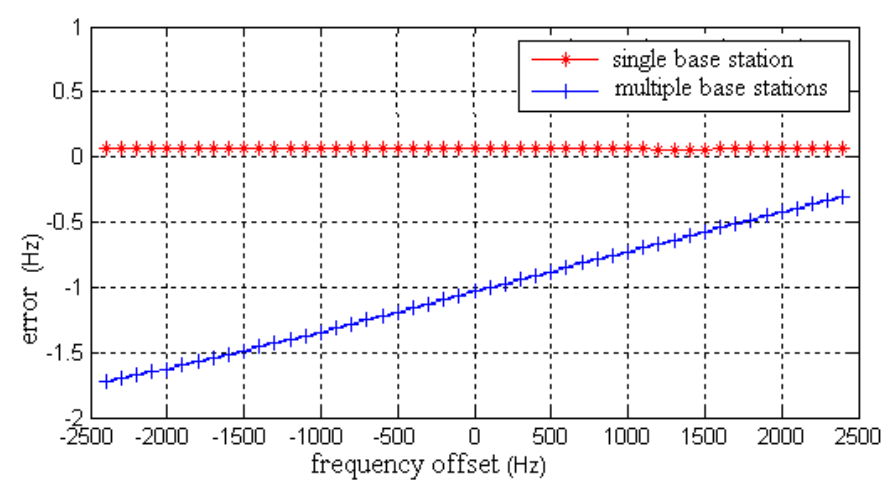

Fig.2.3 result of the composite fractional frequency offset estimation

As it shows, in the range of the frequency offset we set, although the frequency offset estimation error of the composite signal is larger than the single signal, the maximum frequency offset estimation error is still less than $2 \mathrm{~Hz}$, which only accounts for $0.1 \%$ (less than $1 \%$ ) of the sub carrier interval, so we still think regard it as an effective method for complex frequency offset estimation.

2.2. The composite fractional frequency offset under the condition of small delay

The simulation in the section 2.1 is based on the sliding correlated result and the composite frequency offset estimated error. The composite fractional frequency offset estimation under the condition of small time-delay in this section. In the simulation, we assume that the time interval among the three signals is 5 sampling points (0.5us, which is the equivalent of a distance of 150 meters) and the amplitude ratio of different time-delay signals is 2:1:1:1. The sliding correlated results of the composite signals are shown in Figure 2.4. The gray curve represents the three signals auto-correlated results, black curve represents sliding correlated results of the composite signals. For comparison, the composite signal carries a weighted factor with the value of $1 / \sqrt{2}$.

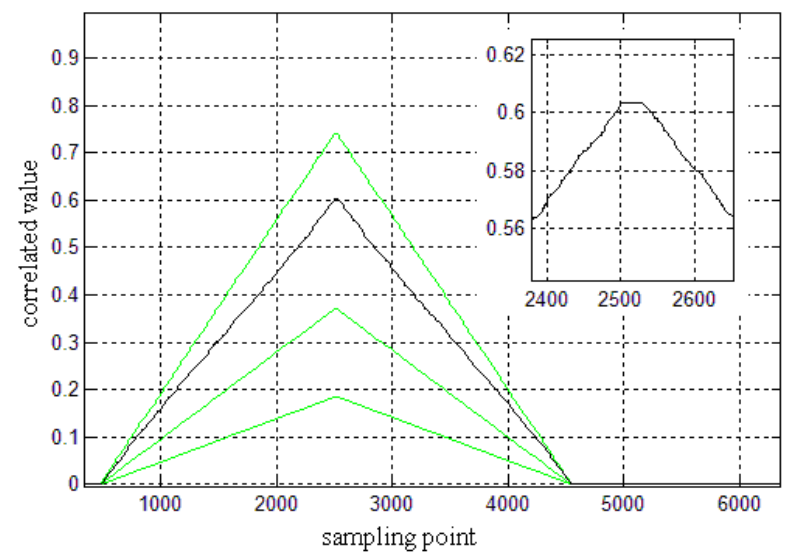

Fig.2.4 the sliding correlated results of the composite signal under the condition of small delay

As it shows, in the case of the less time delay of each signal, the correlated peak of the composite signal is close to each other whether the strength of each signal are the same or not, and the correlated peaks of the composite signal are close proximity, and the peak is close to the strongest single signal correlated peak. Further simulation can also illustrate this issue.

The curve of the composite fractional frequencies offset estimation error of the composite signal with delay of five sample point is shown in Fig.2.5 , in which the horizontal coordinates are represented as fractional inaccuracy and the error of the fractional frequency offset is in the vertical coordinate. The "*" curve represents the result of single signal frequency offset estimation and the "+" curve represents the result of the composite signal frequency offset estimation. 


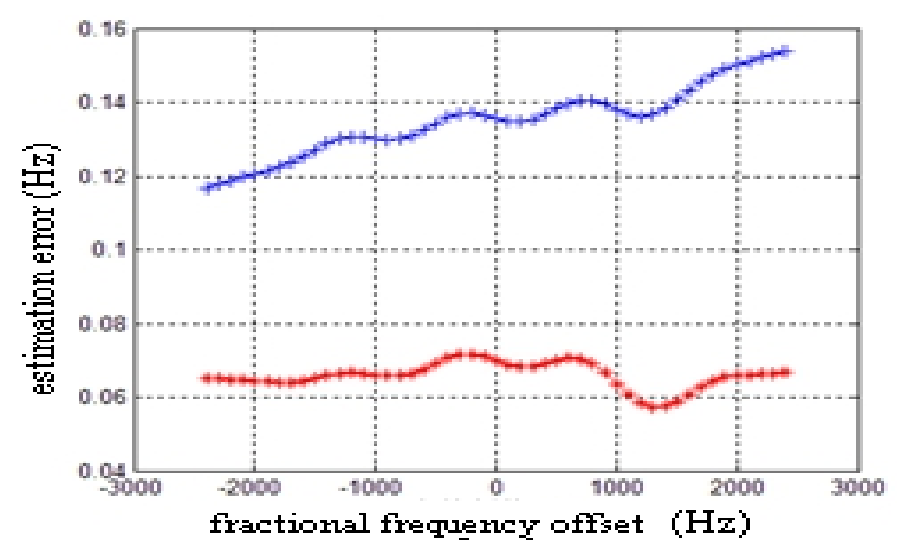

Fig.2.5 the composite frequency offset estimation error with small delayed composite signals

As can be seen from the error curve that the fractional frequency offset of the composite signal has a similar error curve with the single signal in the condition of less time-delay of each signal, just the absolute value of the error is slightly larger than that of the single signal, but still far less than the sub carrier interval of 1\%. From the error curve of frequency offset estimation, it can be seen that the fractional frequency offset estimation error of the single signal and the composite signal is almost the same regardless of the signal intensity of each signal, which also proves that the method is effective for the composite fractional frequency offset estimation.

\section{Performance of the composite fractional frequency offset estimation in AWGN channel}

It is inevitable that the interference from Additive White Gauss Noise (AWGN) exists in the process of signal transmission. The signals from different base stations in the positioning system arrive in the receiver through different paths, which can cause the different AWGN interference. The received signal in a receiver is a composite signal formed by some signals with different time-delays and amplitudes. According to the signal system in which the signal is transmitted by Time Division Duplexing (TDD) technique. The receiver can receive the composite signals from three different base stations at the same time. The composite signal arrives at the receiver in different time and with different AWGN, which keeps changing with the change of the receiver position. So in the process of simulation, it is impossible to simulate the real environment completely.

From the simulation of the upper section, it can be known that the correlated peak and fractional frequency offset estimation error are similar under no AWGN condition. So this paper is aimed at simulation of the correlated peak and fractional frequency offset estimation error under the different amplitude signals and the different signal to noise ratio (SNR) condition. The simulation assumes that the value of the SNR is 10, which is in accordance with the actual situation.

In the simulation, the baseband signal is generated according to the signal system, and then the signal is modulated to $36 \mathrm{MHz}$ intermediate frequency signal which adds different frequency offset. After filtering, both the different weighted time-delay signals and AWGN are superposed to form a multipath composite signal, and then this signal is converted into a zero intermediate frequency multipath signal, which passes through a low-pass filter. At last we slide the signal and correlate with the local synchronization signal, and then look for the relevant peak to calculate the frequency offset. The composite frequency offset estimation error of the mixed signals under AWGN channel is shown in Fig.2.6. 


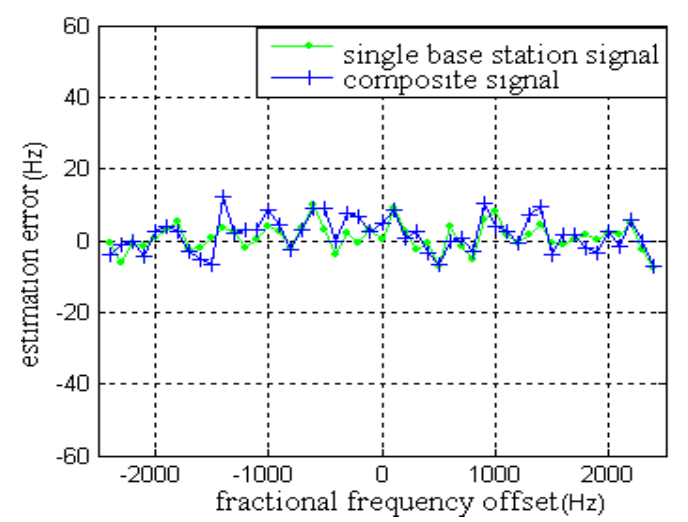

Fig.2.6 curve of the composite frequency offset estimation error under AWGN channel

As Fig.2.6 shows that the curves of the frequency offset estimation error of the composite signal and the single signal are similar in general, but the fluctuation range of frequency is less than $12 \mathrm{~Hz}$, which is close to the $8 \mathrm{~Hz}$ of the single signal estimation error. The curves also prove that the method has a favorable performance of the composite fractional frequency offset estimation under AWGN channel.

\section{Composite integral frequency offset estimation}

In OFDM system, without considering the receiver moving, the integral frequency offset mainly be caused by the aging of the oscillator in the receiver. In the Single Frequency Network (SFN), since the PN sequence after a operation by the Inverse Fast Fourier Transform (IFFT)(that is, OFDM modulation) has still a good self-correlation characteristics. The local perfect synchronous sequence after the integral frequency offset compensation is used to make a correlated operation with the received data stream after the fractional frequency offset compensation. The multiple of the integer multiples frequency offset is constantly changed until the correlation peak are found. When the different time-delay and intensity signals from each base station are received, we can verify whether the method is suitable or not through the simulation of the mixed signals

Fig.2.7 is the simulation figure that the local signal before and after integer frequency offset compensation are made correlated operation with the composite signal. The simulation process is similar to Fig.2.2, but the difference between Fig.2.7 and Fig.2.2 is that the integer frequency offset is added. (a) is the correlated figure before the integral frequency offset compensation, (b) is the correlated figure after the integral frequency offset compensation.

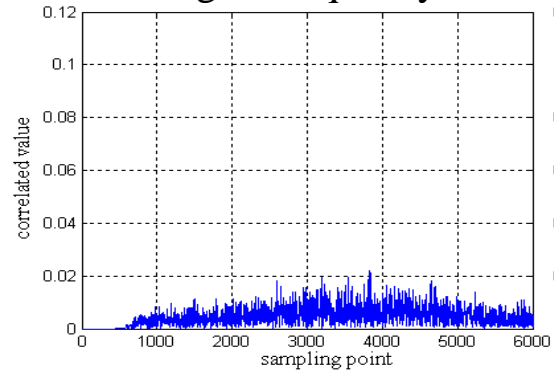

(a)Before compensation

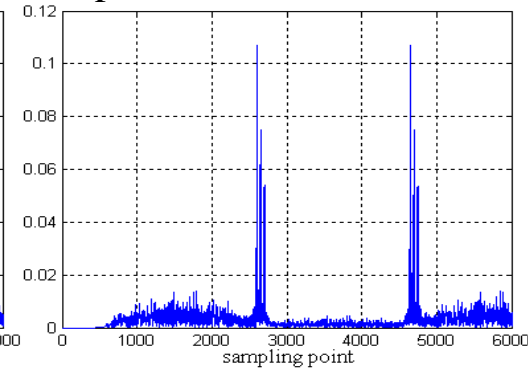

(b)after compensation

Fig.2.7 simulation figure that the local signal correlated operation with the composite signal.

Fig.2.7 shows that the correlation peaks are not found in the correlated results before the integral frequency offset compensation. Two correlated peaks apart from 2048 sampling points occur after the integral frequency offset compensation. The correlated peak value is $10 \mathrm{~dB}$ higher than the correlated average value. The simulation shows that there is no correlated peak between the received signal and the local signal in the absence of integral frequency offset compensation, which also verifies the validity of the composite integral frequency offset estimation.

\section{Conclusion}

In this paper, the estimation algorithm of the integral multiplication frequency and fractional multiplication frequency offset is applied to the frequency offset estimation of composite signal and 
is approved in OFDM system. The algorithm is verified by improved simulation. The simulation results show that the performance of the composite frequency offset estimation under the condition of no white noise interference is slightly lower than that of single signal frequency offset estimation, but the results are far less than 1\% of the sub carrier frequency. While the AWGN interference exists and the value of SNR is greater than $5 \mathrm{~dB}$, the algorithm can achieve frequency offset estimation. The simulation results show that the maximum likelihood fractional frequency offset estimation and the integer frequency offset estimation of the sweeping frequency method can be applied to frequency offset estimation of the composite signal in OFDM system.

\section{Acknowledgements}

This work was financially supported "Research on Integrated Navigation and positioning technology based on digital TV”.

\section{References:}

[1] Classen F, Meyr H. Frequency synchronization algorithms for OFDM systems suitable for communication over frequency selective fading channels. IEEE vehic. Tech. Conf. Stockholm, Sweden, June 1994.

[2] Tufvesson F, Edfors O, Faulkner M. Time and Frequency synchronization for OFDM using PN-sequence preambles. IEEE vehic. Tech. Conf., Amsterdam, the Metherlands, Sept.1999.

[3] Van de Beek J J, Sandell M, Boriesson P O. ML estimation of time and frequency offset in OFDM systems. IEEE Trans. on Signal Processing, 1997,45(7):1800-1805.

[4] Tureli U, Lui H, Zoltowki M O. Blind carrier offset estimation algorithms for DFT-based OFDM systems. IEEE Communications Letters. Apr.1998.

[5] Mao Jianhui, Hei Yong, and Qiao Shushan. "Design of frequency and sampling clock synchronization for CMMB system”[J]. Beijing: micro-computer information,vol.24, No.8-3, 2008.

(in Chinese) .

[6] Luise M, Marselli M, Reggiannini R. Low-complexity blind carrier frequency recovery for OFDM signal over frequency-selective radio channels. IEEE Trans. on Communications, 2002,50(7):1182-1188.

[7] Huang Changjiang, Hu Yonghui, Zhang Huijun, Wu Jianfeng. Research on pseudo- range measurement technique for CMMB system(C). IEEE EFTF/IFC 2013:356-359.

[8] Huang Changjiang, Research on Terrestrial Multimedia Broadcast Positioning Technology Based on CMMB(D). National Time Service Center, Chinese Academy of Sciences,2014.5:87-91. 